Determinação de Substâncias Bioativas em Arilos dos Frutos de Clusia fluminensis Planch. \& Triana

\author{
Mazza, K. E. L.; Santiago, M. C. P. A.; Pacheco, S.; Nascimento, L. S. M.; \\ Braga, E. C. O.; Martins, V. C.; Cunha, C. P.; Godoy, R. L. O.; Borguini, R. G
}

Rev. Virtual Quim., 2019, 11 (1), 3-17. Data de publicação na Web: 21 de agosto de 2018

http://rvq.sbq.org.br

\title{
Determination of Bioactive Compounds in Clusia fluminensis Planch. \& Triana fruit's arils
}

\begin{abstract}
Brazil is considered the country that has the greatest biodiversity on the planet, with many endemic plant species. The phytochemical study, through the separation and characterization of the substances present in plants, can contribute to increase the economy and the survival of plant species such as Clusia fluminensis Planch. \& Triana. This plant, commonly known as abaneiro and mangue-de-praia, belongs to the Clusiaceae family, it is native and endemic from Brazil, found mainly in Bahia, Espírito Santo and Rio de Janeiro States. The objective of this study was to characterize, by High Performance Liquid Chromatography (HPLC), the bioactive compounds presents in the arils of the Clusia fluminensis fruit. For this study, the fruits were collected in Barra da Tijuca and Recreio dos Bandeirantes neighborhoods, in the city of Rio de Janeiro - Brazil. The chromatographic analyzes were performed using the extracts of arils with seeds of the fruit. It was identified some phenolic compounds as protocatechuic acid, 4-hydroxybenzoic acid and 4-hydroxycinnamic ( $p$-coumaric) acid, flavonoid rhamnetina (7-methoxyquercetin) and some carotenoids as lutein, zeaxanthin and $\beta$-cryptoxanthin. A low content of vitamin $C$ in the fruit aryls was quantified $(3.77 \mathrm{mg} .100 \mathrm{~g}-1)$. The high concentration of zeaxanthin $\left(823 \mu \mathrm{g} . \mathrm{g}^{-1}\right)$ in the arils was highlighted, once it is a carotenoid that plays an important role in the macula, with content 206 times higher than the one found in corn in natura. Few scientific papers published are related to these fruits. Therefore, the results support a better understanding of the chemical composition of this species, describing the occurrence of bioactive compounds in Clusia fluminensis fruit's arils.
\end{abstract}

Keywords: Clusiaceae, vitamin C, carotenoids, flavonoids, phenolic acids.

Resumo

O Brasil é considerado o país que detém a maior biodiversidade do planeta, muitas de suas espécies botânicas são endêmicas. $O$ estudo fitoquímico, através da separação e caracterização das substâncias presentes nas plantas, pode contribuir para o aumento da importância econômica e a sobrevivência destas espécies botânicas como a Clusia fluminensis Planch. \& Triana. Esta planta, conhecida popularmente como abaneiro e mangue-de-praia, pertence à família Clusiaceae, é nativa e endêmica do Brasil, sendo encontrada principalmente nos estados da Bahia, Espírito Santo e Rio de Janeiro. 0 objetivo deste trabalho foi caracterizar, por Cromatografia Líquida de Alta Eficiência (CLAE), as substâncias bioativas presentes nos arilos do fruto de Clusia fluminensis. Para este estudo, os frutos foram coletados nos bairros Barra da Tijuca e Recreio dos Bandeirantes, na cidade do Rio de Janeiro - RJ. As análises cromatográficas foram realizadas a partir dos extratos dos arilos com sementes dos frutos. Foram identificados compostos fenólicos como os ácidos protocatecuico, 4hidroxibenzoico e 4-hidroxicinâmico ( $p$-cumárico), o flavonoide rhamnetina (7-metóxiquercetina) e os carotenoides luteína, zeaxantina e $\beta$-criptoxantina. Foi quantificado um baixo teor de vitamina $C$ nos arilos do fruto $(3,77 \mathrm{mg} .100 \mathrm{~g}-1)$. Destaca-se a elevada concentração de zeaxantina $\left(823 \mu \mathrm{g} \cdot \mathrm{g}^{-1}\right)$, carotenoide que desempenha um importante papel na mácula, com teor 206 vezes superior ao milho verde in natura. Poucos trabalhos científicos publicados são relacionados a esses frutos. Por isso, os resultados contribuem para uma melhor compreensão da composição química desta espécie, ao descrever a ocorrência de substâncias bioativas nos arilos dos frutos de Clusia fluminensis.

Palavras-chave: Clusiaceae, vitamina C, carotenoides, flavonoides, ácidos fenólicos.

\footnotetext{
* Embrapa Agroindústria de Alimentos, Laboratório de Cromatografia Líquida (CLAE), 23020-470, Rio de Janeiro - RJ, Brasil.

M karenelbert@gmail.com

DOI: $\underline{10.21577 / 1984-6835.20190002}$
} 


\title{
Determinação de Substâncias Bioativas em Arilos dos Frutos de Clusia fluminensis Planch. \& Triana
}

\author{
Karen E. L. Mazza, ${ }^{\mathrm{a}, *}$ Manuela C. P. A. Santiago, ${ }^{\mathrm{b}}$ Sidney Pacheco, ${ }^{\mathrm{b}}$ \\ Luzimar S. M. do Nascimento, ${ }^{b}$ Elaine C. O. Braga, ${ }^{a}$ Víctor C. Martins, ${ }^{c}$ \\ Carolina P. da Cunha, ${ }^{d}$ Ronoel L. O. Godoy, ${ }^{b}$ Renata G. Borguini ${ }^{b}$
}

a Universidade Federal do Rio de Janeiro, Instituto de Química, 21941-909, Cidade Universitária-Rio de Janeiro- RJ, Brasil.

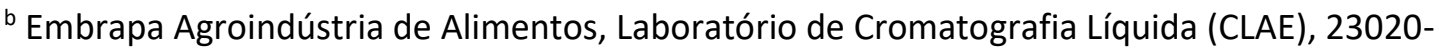
470, Rio de Janeiro-RJ, Brasil.

c Universidade Federal Rural do Rio de Janeiro, Instituto de Tecnologia, Departamento de Tecnologia de Alimentos, 23890-000, Seropédica-RJ, Brasil.

d Universidade Federal Rural do Rio de Janeiro, Instituto de Ciências Exatas, Departamento de Química, 23890-000, Seropédica-RJ, Brasil.

\section{* karenelbert@gmail.com}

Recebido em 17 de novembro de 2016. Aceito para publicação em 15 de junho de 2018

1. Introdução

2. Objetivo

3. Material e Métodos

3.1. Solventes, reagentes e padrões analíticos

3.2. Procedência das amostras

3.3. Análise de ácido ascórbico (Vitamina C)

3.4. Análise de ácidos fenólicos

3.5. Análise de flavonoides

3.6. Análise de carotenoides

4. Resultados e Discussão

4.1. Ácido ascórbico (Vitamina C)

4.2. Ácidos fenólicos

4.3. Flavonoides

4.4. Carotenoides

5. Conclusão

6. Referências Bibliográficas

\section{Introdução}

A espécie Clusia fluminensis Planch. \& Triana, pertencente à família Clusiaceae e de sinônimo heterotípico Clusia ildefonsiana A. Rich. ex Planch. \& Triana, consiste em uma espécie nativa e endêmica do Brasil, distribuída principalmente nos estados da Bahia, Espírito Santo e Rio de Janeiro, tendo 
por domínio geográfico a Mata Atlântica. Conhecida popularmente como abaneiro ou mangue-da-praia, esta espécie é utilizada para fins de ornamentação, apresentando-se em forma de arbustos ou árvores e geralmente podendo alcançar de 2 a 3 metros de altura..$^{1,2,3,4}$

Os frutos desta espécie apresentam coloração esverdeada, possuindo em seu interior cápsulas contendo arilos, membranas de cor alaranjada onde as sementes encontram-se dispersas ${ }^{5}$. Destacam-se os arilos (Figura 1) pelo alto consumo por animais silvestres e pela coloração característica da presença de carotenoides.

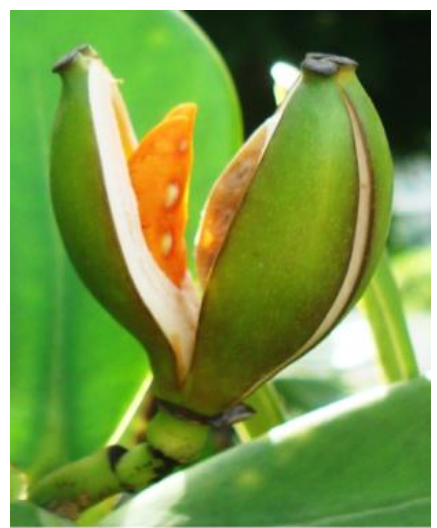

Figura 1. Frutos maduros de Clusia fluminensis, com destaque para os arilos. Foto: Sidney Pacheco.

O Brasil é detentor da maior biodiversidade do planeta, com muitas espécies endêmicas, como os frutos abacaxi (Ananas comosus L. Merril), caju (Anacardium occidentale L.) e açaí (Euterpe oleraceae Mart.), os quais que possuem relevância na economia global principalmente na indústria alimentícia. Desta forma, o estudo de outros frutos originários da flora brasileira torna-se importante pela exploração da diversidade observada no país e pelo aumento de informações sobre a composição química dos mesmos, possibilitando a aplicação para diferentes finalidades nos segmentos da indústria química, farmacêutica, alimentícia, cosmética, entre outros. ${ }^{6}$

Com o intuito de separar, identificar e quantificar as substâncias provenientes de uma mistura, podem ser utilizadas determinadas técnicas, como a cromatografia, cuja aplicação na área da química de produtos naturais auxilia na separação e caracterização das substâncias presentes nas plantas. ${ }^{7,8}$

Em seu estudo com $C$. fluminensis, Silva isolou os terpenos 3-oxo-friedelina e epifriedelinol dos extratos hexânicos de folhas e caules e ainda a benzofenona clusianona e o terpenoide lanosterol nos extratos hexânicos das flores. ${ }^{9}$ Quanto aos frutos de $C$. fluminensis, poucos relatos foram encontrados na literatura sobre os fitoquímicos dessa espécie.

Silva \& Paiva (2012) efetuaram a quantificação de flavonoides totais expressos em rutina por espectrofotometria para os extratos metanólico e acetônico dos frutos de Clusia fluminensis e avaliaram sua atividade antioxidante com o emprego de DPPH. No entanto, não foi realizada a identificação destes flavonoides, mencionando somente a ocorrência de flavonas e flavonóis. ${ }^{3}$

\section{Objetivo}

Este trabalho teve por objetivo caracterizar as substâncias bioativas presentes nos arilos do fruto de Clusia fluminensis por Cromatografia Líquida de Alta Eficiência (CLAE).

\section{Material e Métodos}

\subsection{Solventes, reagentes e padrões} analíticos

Os solventes acetato de etila $\left(\mathrm{C}_{4} \mathrm{H}_{8} \mathrm{O}_{2}\right)$, acetonitrila $\left(\mathrm{C}_{2} \mathrm{H}_{3} \mathrm{~N}\right)$, acetona $\left(\mathrm{C}_{3} \mathrm{H}_{6} \mathrm{O}\right)$, ácido acético $\left(\mathrm{C}_{2} \mathrm{H}_{4} \mathrm{O}_{2}\right)$, ácido fórmico $\left(\mathrm{CH}_{2} \mathrm{O}_{2}\right)$, éter de petróleo, éter etílico $\left(\mathrm{C}_{4} \mathrm{H}_{10} \mathrm{O}\right)$, éter metil terc-butílico $\left(\mathrm{C}_{5} \mathrm{H}_{12} \mathrm{O}\right)$ e metanol $\left(\mathrm{CH}_{4} \mathrm{O}\right)$ foram adquiridos da Tedia (Brasil). A água ultrapurificada foi obtida através do sistema de purificação de água Milli- $Q^{\circ}$ Gradient $10 \mathrm{~A}$ Millipore ${ }^{\bullet}$ e Elix $/$ RiOs $^{\text {TM }} 5$ (EUA). 
Foram utilizados ainda o ácido clorídrico $(\mathrm{HCl})$, ácido fosfórico $\left(\mathrm{H}_{3} \mathrm{PO}_{4}\right)$ e ácido sulfúrico $\left(\mathrm{H}_{2} \mathrm{SO}_{4}\right)$ Tedia ${ }^{\circ}$ (Brasil), com grau P.A.; celite 545 Tedia $^{\circ}$ (Brasil); cloreto de sódio $(\mathrm{NaCl})$ Dinâmica (Brasil), sulfato de sódio anidro $\left(\mathrm{Na}_{2} \mathrm{SO}_{4}\right)$, hidróxido de sódio $(\mathrm{NaOH})$, hidróxido de potássio (KOH) e di-butilhidroxitolueno ou BHT $\left(\mathrm{C}_{15} \mathrm{H}_{24} \mathrm{O}\right)$ Spectrum (EUA).

Para identificação e quantificação dos analitos, foram empregados padrões de ácidos fenólicos (ácidos gálico, protocateico, 4-hidroxibenzoico, cafeico, 4-hidroxicinâmico, ferúlico e sinápico), de flavonoides (epicatequina, rutina, rhamnetina, narirutina, mirecetina, diosmina, hesperidina, quercetina e kaempferol) e de ácido L-ascórbico, SigmaAldrich ${ }^{\circ}$ (EUA). Os padrões de carotenoides foram obtidos segundo metodologia de Pacheco $(2013)^{10}$, por isolamento a partir de matrizes vegetais no Laboratório de Cromatografia Líquida de Alta Eficiência (CLAE) na Embrapa Agroindústria de Alimentos (Rio de Janeiro, Brasil), com pureza superior a $95 \%$.

\subsection{Procedências das amostras}

Foram coletados cerca de $40 \mathrm{~g}$ de frutos maduros de $C$. fluminensis nos bairros da Barra da Tijuca $\left(22^{\circ} 58^{\prime} 13.886^{\prime \prime} \quad \mathrm{S}\right.$; $43^{\circ} 23^{\prime} 10.759^{\prime \prime}$ W) e do Recreio dos Bandeirantes $\left(23^{\circ} 00^{\prime} 58.7^{\prime \prime S}\right.$; 4330'59.4"W) localizados na cidade do Rio de Janeiro, em junho e julho de 2014. Após seleção, foram separados manualmente os arilos com sementes da casca, sendo a casca descartada. Foi avaliada a presença das seguintes substâncias: vitamina C, ácidos fenólicos, flavonoides e carotenoides. Foi pesado, em duplicata, cerca de $1 \mathrm{~g}$ dos arilos com as sementes, devido à dificuldade de separação da semente. Procedeu-se a análise de vitamina $\mathrm{C}$ no mesmo dia, devido à labilidade do analito, sendo o restante armazenado em frascos de vidro âmbar e armazenado em freezer a $-18{ }^{\circ} \mathrm{C}$ até o momento das demais análises (flavonoides, ácidos fenólicos e carotenoides). Todas as análises foram realizadas no Laboratório de Cromatografia Líquida da Embrapa Agroindústria de Alimentos, acreditado pelo INMETRO de acordo com a ISO 17025 para análises de carotenoides.

\subsection{Análise de ácido ascórbico (Vitamina} C)

A determinação de ácido ascórbico (vitamina C) baseou-se no método de Rosa e colaboradres. ${ }^{11} \mathrm{~A}$ análise cromatográfica foi realizada em um cromatógrafo líquido de alta eficiência Waters ${ }^{\circ}$ modelo Alliance 2690/5, detector de arranjo de fotodiodos Waters ${ }^{\circ}$ modelo 2996, software Empower ${ }^{\circ}$ e coluna de troca iônica Aminex HPX-87H BIO-RAD (300 x $7,8 \mathrm{~mm}$ ), com as seguintes condições cromatográficas: temperatura da coluna de 30 ${ }^{\circ} \mathrm{C}$; temperatura do injetor de $15{ }^{\circ} \mathrm{C}$; fluxo de $0,7 \mathrm{~mL} \cdot \mathrm{min}^{-1}$ em modo de eluição isocrático com solução aquosa de ácido sulfúrico 0,05 mol. $\mathrm{L}^{-1}$; tempo de corrida de $10 \mathrm{~min}$; e volume de injeção de $20 \mu \mathrm{L}$. A quantificação da vitamina $C$ foi realizada por padronização externa com curva analítica construída a partir de soluções padrão, em comprimento de onda de $242,6 \mathrm{~nm}$.

\subsection{Análise de ácidos fenólicos}

Foi utilizado o método descrito por Mattila \& Kumpulainen, ${ }^{12}$ com a obtenção de 3 frações: ácidos fenólicos livres (AFL), hidrólise básica e hidrólise ácida, em que a soma dos teores hidrolisados constituem os ácidos fenólicos totais (AFT). Para a análise, foi utilizado um cromatógrafo líquido de alta eficiência Waters Alliance modelo 2690/5, detector de arranjo de fotodiodos Waters ${ }^{\circ}$ modelo 2996 (varredura 210 a $600 \mathrm{~nm}$ ), software Empower ${ }^{\circ}$ e coluna Thermo BDS HYPERSIL $C_{18}(100 \times 4,6 \mathrm{~mm} ; 2,4 \mu \mathrm{m})$. Utilizouse as seguintes condições cromatográficas: temperatura da coluna igual a $30^{\circ} \mathrm{C}$; fluxo de 1,0 a $1,2 \mathrm{~mL} \cdot \mathrm{min}^{-1} \mathrm{em}$ modo de eluição em gradiente de solução aquosa de ácido fosfórico a 1,5 mL.L L $^{-1}$ (fase móvel A) e 
acetonitrila (fase móvel B), como exibido na Tabela 1; tempo de corrida de $28 \mathrm{~min}$; e volume de injeção de $10 \mu \mathrm{L}$.

Os ácidos fenólicos foram identificados por comparação dos tempos de retenção e espectros de absorção na região do Ultravioleta/Visível (UV-Vis) dos picos observados nos cromatogramas da amostra e da mistura de padrões de ácidos fenólicos.

A quantificação de ácidos fenólicos foi realizada por padronização externa com curva analítica construída a partir de soluções padrão, em comprimentos de onda de $270 \mathrm{~nm}$ e $310 \mathrm{~nm}$.

Tabela 1. Gradiente de eluição da fase móvel para separação dos ácidos fenólicos

\begin{tabular}{cccc}
\hline Tempo & Fluxo $\left(\mathbf{m L} \cdot \mathbf{m i n}^{-1}\right)$ & Fase A (\%) & Fase B (\%) \\
\hline 0,00 & 1,0 & 95,0 & 5,0 \\
6,00 & 1,0 & 95,0 & 5,0 \\
12,00 & 1,2 & 88,0 & 12,0 \\
18,00 & 1,2 & 80,0 & 20,0 \\
20,00 & 1,2 & 50,0 & 50,0 \\
24,00 & 1,2 & 50,0 & 50,0 \\
25,00 & 1,2 & 95,0 & 5,0 \\
28,00 & 1,0 & 95,0 & 5,0 \\
\hline * Fase A - ácido fosfórico 1,5 $\mathrm{mL}^{-1}$ em água; Fase B - acetonitrila
\end{tabular}

* Fase $\mathrm{A}$ - ácido fosfórico $1,5 \mathrm{~mL} \cdot \mathrm{L}^{-1}$ em água; Fase $\mathrm{B}$ - acetonitrila

\subsection{Análise de flavonoides}

A extração de flavonoides foi realizada segundo o método de Klump (2001). ${ }^{13}$ Procedeu-se a análise em um cromatógrafo líquido de alta eficiência Waters ${ }^{\circ}$ Alliance modelo 2690/5, detector de arranjo de fotodiodos Waters modelo 2996 (varredura 210 a $600 \mathrm{~nm}$ ), software Empower e coluna Thermo BDS HYPERSIL $C_{18}(100 \times 4,6 \mathrm{~mm} ; 2,4$ $\mu \mathrm{m})$. As condições cromatográficas foram: temperatura da coluna em $45^{\circ} \mathrm{C}$; temperatura do injetor em $15{ }^{\circ} \mathrm{C}$; fluxo de $1 \mathrm{~mL} \cdot \mathrm{min}^{-1}$ em modo de eluição gradiente de solução aquosa de ácido fórmico a $10 \mathrm{~mL} . \mathrm{L}^{-1}$ (fase móvel $A$ ) e acetonitrila (fase móvel B), conforme a Tabela 2; tempo de corrida de 28 min e volume de injeção de $10 \mu \mathrm{L}$. A identificação foi realizada por comparação dos tempos de retenção e espectros de UV-Vis de uma mistura de padrões de flavonoides injetada sob as mesmas condições cromatográficas da amostra.

Tabela 2. Gradiente de eluição da fase móvel para separação dos flavonoides

\begin{tabular}{ccc}
\hline Tempo & Fase A (\%) & Fase B (\%) \\
\hline 0,00 & 95,0 & 5,0 \\
15,00 & 95,0 & 5,0 \\
23,00 & 88,0 & 12,0 \\
24,00 & 80,0 & 20,0 \\
28,00 & 50,0 & 50,0 \\
\hline
\end{tabular}

* Fase A - ácido fórmico 1 \% em água; Fase B - acetonitrila 


\subsection{Análise de carotenoides}

A extração de carotenoides totais e a posterior reação de saponificação foram realizadas segundo a metodologia descrita por Rodriguez-Amaya. ${ }^{14} \mathrm{~A}$ determinação do perfil dos carotenoides foi feita segundo a metodologia de Pacheco et al. (2014) usando cromatógrafo líquido de alta eficiência Waters Modular composto por bomba 600, injetor automático 717 plus, com detector de arranjo de fotodiodos Waters modelo 996 (varreadura 300 a $500 \mathrm{~nm}$ ), software Empower e coluna YMC Carotenoid S-3 (250 $x 4,6 \mathrm{~mm} ; 3 \mu \mathrm{m})$, nas seguintes condições cromatográficas: temperatura da coluna de 33 ${ }^{\circ} \mathrm{C}$; fluxo de $0,8 \mathrm{~mL} \cdot \mathrm{min}^{-1} \mathrm{em}$ modo de eluição gradiente de metanol (fase móvel $A$ ) e étermetil-terc-butílico (fase móvel B), conforme apresentado na Tabela 3; tempo de corrida de 28 min e volume de injeção de $15 \mu \mathrm{L}$. A identificação foi realizada por comparação dos tempos de retenção e espectros de UV-Vis com uma mistura de padrões de carotenoides previamente isolados. A quantificação dos carotenoides foi determinada por padronização externa, através da construção de curvas analíticas a partir das áreas obtidas em comprimento de onda de $450 \mathrm{~nm}$ de soluções padrão de carotenoides.

Tabela 3. Gradiente de eluição das fases móveis para separação dos carotenoides

\begin{tabular}{ccc}
\hline Tempo & Fase A (\%) & Fase B (\%) \\
\hline 0,00 & 80,0 & 20,0 \\
0,50 & 75,0 & 25,0 \\
15,00 & 15,0 & 85,0 \\
15,05 & 10,0 & 90,0 \\
16,50 & 10,0 & 90,0 \\
16,55 & 80,0 & 20,0 \\
28,00 & 80,0 & 20,0 \\
\hline
\end{tabular}

* Fase A - metanol; Fase B - éter metil terc-butílico

\section{Resultados e Discussão}

\section{1. Ácido ascórbico}

A concentração média de vitamina $C$ nos arilos dos frutos de $C$. fluminensis foi de $3,77 \pm$ $0,82 \mathrm{mg} \cdot 100 \mathrm{~g}^{-1}$. Este valor é inferior ao de frutos considerados fontes desta substância, como por exemplo, a laranja pêra $(53,7$ mg.100 g ${ }^{-1}$ ) e acerola $\left(941,4 \mathrm{mg} .100 \mathrm{~g}^{-1}\right),{ }^{16}$ cujos teores são respectivamente 14 e 249 vezes superior ao dos arilos deste fruto. $\mathrm{O}$ teor encontrado para a Clusia fluminensis foi similar ao descrito para outros frutos de importância econômica relevante, como a maçã cv. Fuji $\left(2,4 \mathrm{mg} \cdot 100 \mathrm{~g}^{-1}\right)$, a pera cv. Williams (2,8 mg.100 g $\left.{ }^{-1}\right)$, o pêssego cv. Aurora (3,3 mg.100 g $\mathrm{g}^{-1}$ ) e a uva cv. Itália (3,3 mg.100 $\left.\mathrm{g}^{-1}\right)^{16}$, conforme demonstrado Tabela 4 . 
Tabela 4. Comparação entre os teores de vitamina $C$ dos arilos dos frutos de Clusia fluminensis com outros frutos comestíveis, em mg.100g-1

\begin{tabular}{|c|c|}
\hline & Teor de vitamina $\mathrm{C}\left(\mathrm{mg} \cdot 100 \mathrm{~g}^{-1}\right)$ \\
\hline Acerola (Malpighia glabra L.) $)^{16}$ & 941,4 \\
\hline Laranja pêra (Citrus sinensis) $)^{16}$ & 53,7 \\
\hline Clusia fluminensis & 3,77 \\
\hline Uva cv. Itália (Vitis vinífera L.) $)^{16}$ & 3,30 \\
\hline Pêssego cv. Aurora (Prunus persica L.) ${ }^{16}$ & 3,30 \\
\hline Pêra cv. Williams (Pyrus communis L.) ${ }^{16}$ & 2,80 \\
\hline Maçã cv. Fuji (Malus domestica) ${ }^{16}$ & 2,40 \\
\hline
\end{tabular}

Fonte: Taco (2011). ${ }^{16} \mathrm{~A}$ equação da reta obtida pela curva analítica de ácido ascórbico foi: $\mathrm{y}=$ $1,90 * 10^{6} x-1,16 * 10^{4}$, com coeficiente de determinação $\left(r^{2}\right)$ de 0,9998

\section{2. Ácidos fenólicos}

Foi detectada a presença dos ácidos protocatecuico, 4-hidroxibenzoico e 4hidroxicinâmico (ácido $p$-cumárico), em todas as frações analisadas: ácidos fenólicos livres, hidrólise básica e hidrólise ácida. As Figuras 2, 3,4 e 5 consistem nos cromatogramas, respectivamente, da solução padrão de ácidos fenólicos e das três frações obtidas a partir dos arilos do fruto.

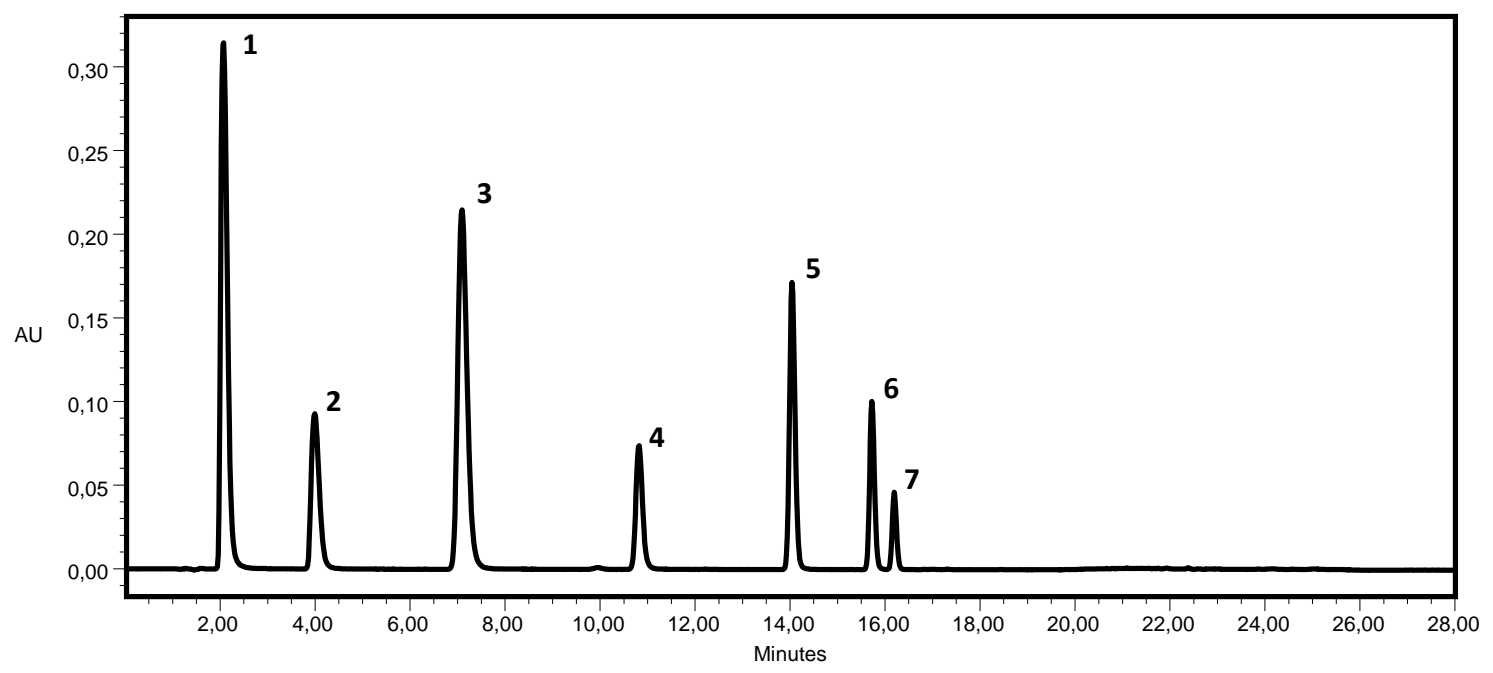

Figura 2. Cromatograma, por CLAE-DAD, da solução padrão dos seguintes ácidos fenólicos: (1) gálico $\left(t_{r}=2,08 \mathrm{~min}\right)$; (2) protocatecuico $\left(t_{r}=4,03 \mathrm{~min}\right)$; (3) 4-hidroxibenzoico $\left(t_{r}=7,21 \mathrm{~min}\right)$; (4) cafeico ( $\left.t_{r}=10,82 \mathrm{~min}\right)$; (5) 4-hidroxicinâmico ( $t_{r}=13$,96 min); (6) ferúlico ( $\left.t_{r}=15,69 \mathrm{~min}\right)$;

(7) sinápico $\left(t_{r}=16,15 \mathrm{~min}\right)$ 


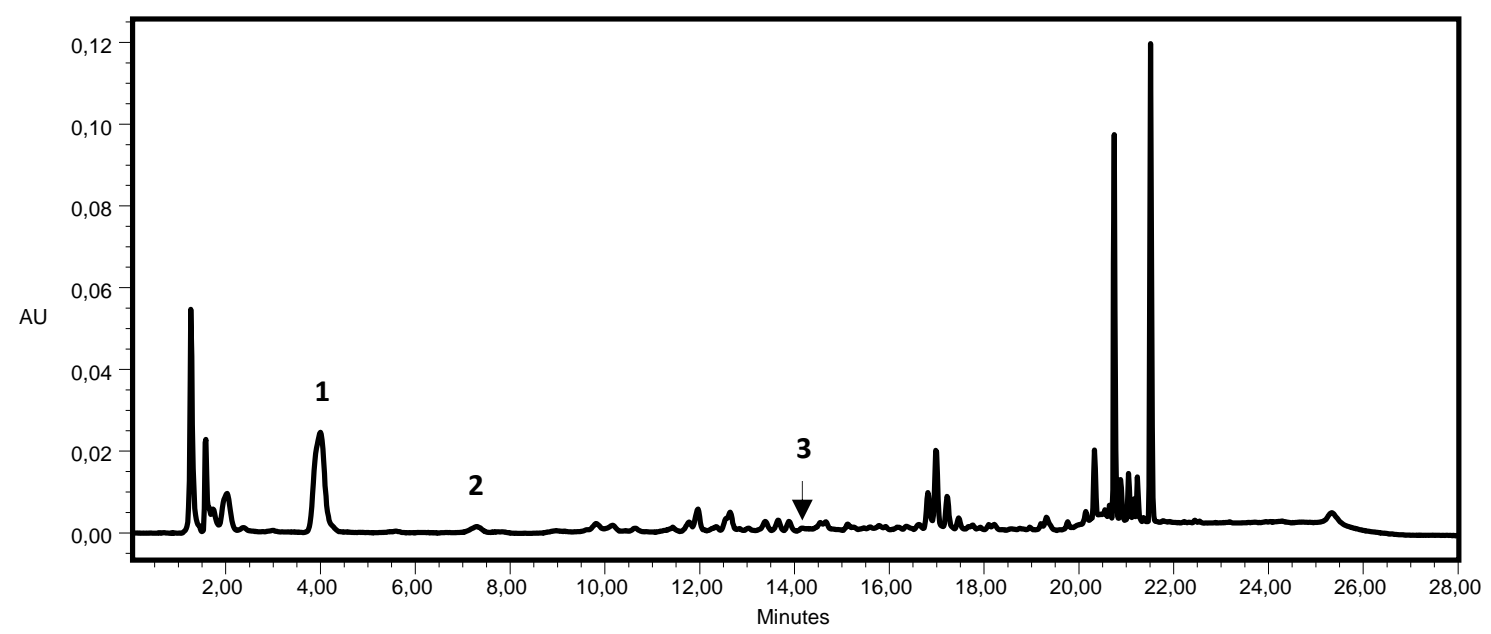

Figura 3. Cromatograma, por CLAE-DAD, referente à fração de ácidos fenólicos livres obtida a partir dos arilos de $C$. fluminensis: (1) ácido protocatecuico ( $t_{r}=3,99 \mathrm{~min}$ ); (2) ácido 4hidroxibenzoico $\left(t_{r}=7,29 \mathrm{~min}\right)$; (3) ácido 4-hidroxicinâmico $\left(t_{r}=14,16 \mathrm{~min}\right)$

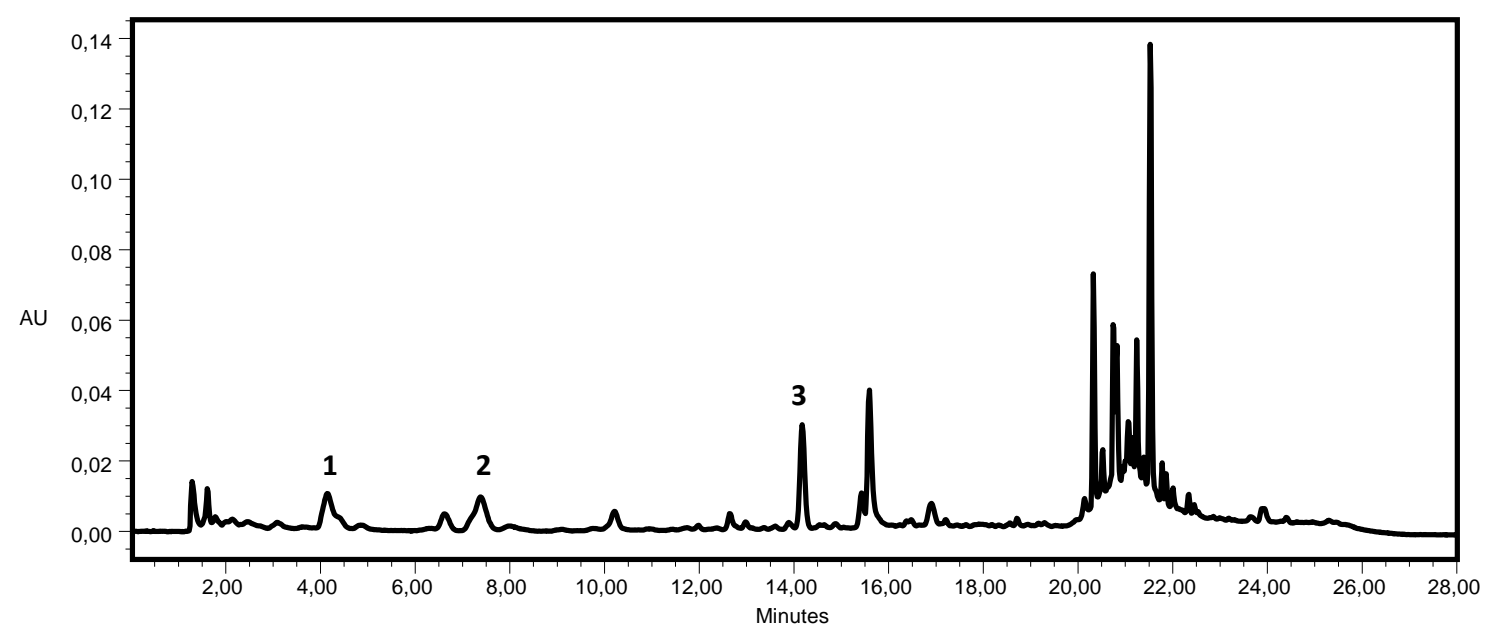

Figura 4. Cromatograma, por CLAE-DAD, referente à fração de ácidos fenólicos após hidrólise básica do extrato dos arilos de $C$. fluminensis: (1) ácido protocatecuico $\left(t_{r}=4,15 \mathrm{~min}\right)$; (2) ácido 4-hidroxibenzoico ( $t_{r}=7,38 \mathrm{~min}$ ); (3) ácido 4-hidroxicinâmico ( $t_{r}=14,17 \mathrm{~min}$ ) 


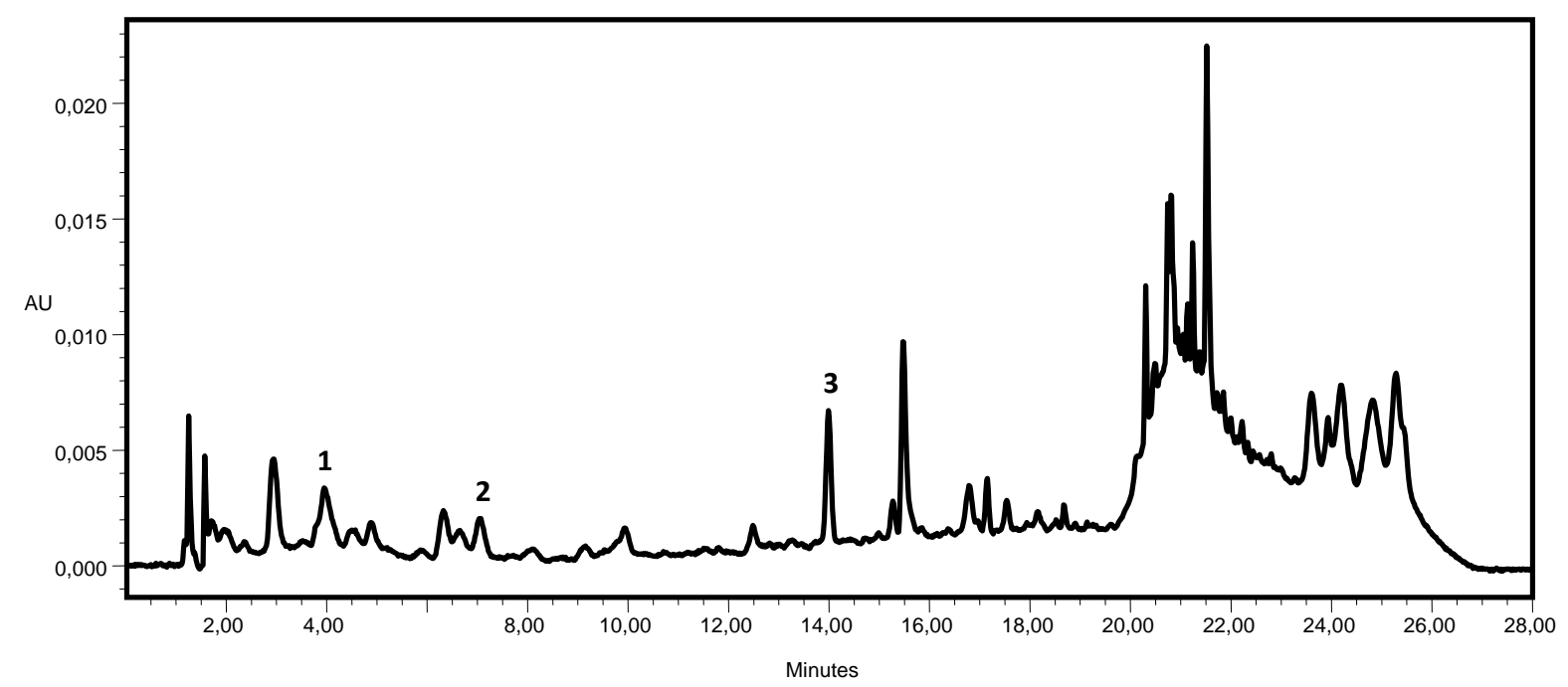

Figura 5. Cromatograma, por CLAE-DAD, referente à fração de ácidos fenólicos após hidrólise ácida do extrato dos arilos de $C$. fluminensis: (1) ácido protocatecuico $\left(t_{\mathrm{r}}=4,12 \mathrm{~min}\right.$ ); (2) ácido 4-hidroxibenzoico $\left(t_{r}=7,22 \mathrm{~min}\right)$; (3) ácido 4-hidroxicinâmico $\left(t_{r}=14,07 \mathrm{~min}\right)$

A comparação entre os tempos de retenção obtidos com a solução padrão e os tempos de retenção obtidos nas amostras (Tabela 5), e entre os espectros de UV/Vis dos picos detectados (Figura 6), possibilitaram a confirmação da identificação das substâncias encontradas (Figura 7).

Tabela 5. Comparação entre os tempos de retenção dos ácidos fenólicos da solução padrão e das frações provenientes do extrato de $\mathrm{C}$. fluminensis

\begin{tabular}{ccccc}
\hline Ácido & $\begin{array}{c}\mathrm{t}_{\mathrm{R}} \text { padrão } \\
(\mathrm{min})\end{array}$ & $\begin{array}{c}\mathrm{t}_{\mathrm{R}} \text { amostra } \\
\text { AFL }(\min )\end{array}$ & $\begin{array}{c}\mathrm{t}_{\mathrm{R}} \text { amostra } \\
\mathrm{HB}(\mathrm{min})\end{array}$ & $\begin{array}{c}\mathrm{t}_{\mathrm{R}} \text { amostra } \\
\mathrm{HA}(\mathrm{min})\end{array}$ \\
\hline Protocatecuico & 4,03 & 3,99 & 4,15 & 4,12 \\
4-hidroxibenzoico & 7,21 & 7,29 & 7,38 & 7,22 \\
4-hidroxicinâmico & 13,96 & 14,16 & 14,17 & 14,07 \\
\hline
\end{tabular}
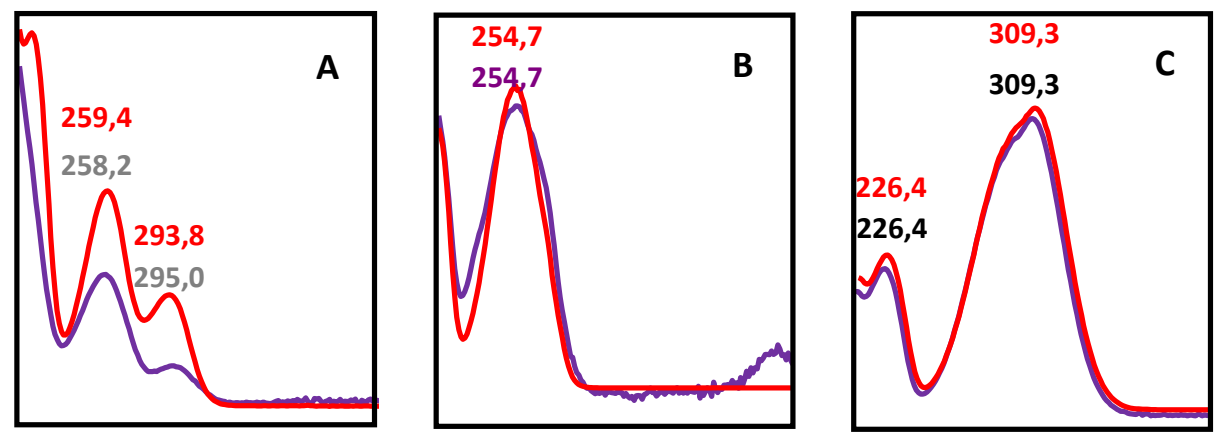

Figura 6. Sobreposição dos espectros de UV/Vis das substâncias identificadas na amostra com os espectros dos padrões: (A) ácido protocatecuico; (B) ácido 4-hidroxibenzoico; (C) ácido 4hidroxicinâmico 


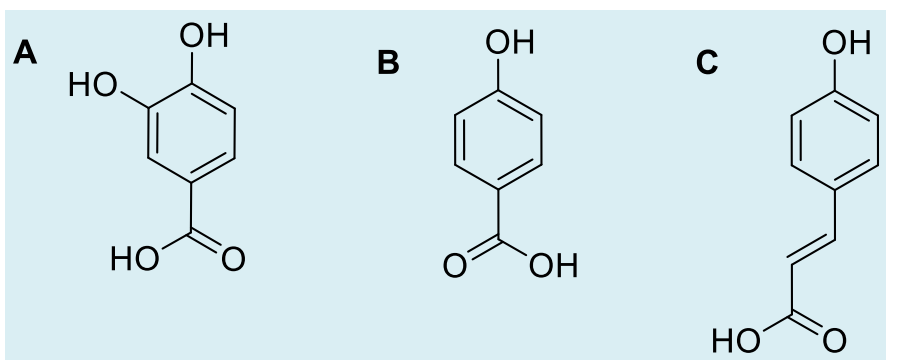

Figura 7. Estruturas químicas dos ácidos: (A) protocatecuico; (B) 4-hidroxibenzoico; (C) 4hidroxicinâmico

Após a identificação, foi realizada uma curva analítica dos ácidos fenólicos identificados, sendo expostos na Tabela 6 as equações da reta e os coeficientes de determinação $\left(r^{2}\right)$ para cada analito.

Tabela 6. Equação da reta e coeficiente de determinação obtidos por meio de curva analítica com padrões de ácidos fenólicos

\begin{tabular}{ccc}
\hline Analito de interesse & Equação da reta & $\mathbf{r}^{\mathbf{2}}$ \\
\hline Ácido Protocatecuico & $\mathrm{y}=2,70^{*} 10^{4} x-7,59 * 10^{3}$ & 0,9994 \\
Ácido $\boldsymbol{p}$-hidroxibenzoico & $\mathrm{y}=3,62 * 10^{4} x-1,54 * 10^{4}$ & 0,9994 \\
Ácido $\boldsymbol{p}$-hidroxicinâmico & $\mathrm{y}=2,61 * 10^{4} x-9,26 * 10^{3}$ & 0,9995 \\
\hline
\end{tabular}

Os teores determinados dos ácidos fenólicos livres e totais presentes nos arilos dos frutos de Clusia fluminensis estão dispostos na Tabela 7.

Tabela 7. Teores, em $\mu \mathrm{g} \cdot \mathrm{g}^{-1}$, dos ácidos fenólicos identificados nos arilos dos frutos de $C$. fluminensis

\begin{tabular}{ccc}
\hline Ácido fenólico & AFL $\left(\boldsymbol{\mu g} \cdot \mathbf{g}^{-1}\right)$ & AFT $\left(\boldsymbol{\mu g} \cdot \mathbf{g}^{-1}\right)$ \\
\hline Protocatecuico & $115,14 \pm 58,23$ & $55,82 \pm 12,23$ \\
$\boldsymbol{p}$-hidroxibenzoico & $11,43 \pm 0,32$ & $16,76 \pm 1,06$ \\
$\boldsymbol{p}$-hidroxicinâmico & $5,97 \pm 0,41$ & $49,03 \pm 12,69$ \\
\hline
\end{tabular}

O ácido fenólico majoritário entre os detectados foi o ácido protocatecuico, que apresentou alta concentração mesmo na fração $\operatorname{AFL}\left(115,14 \mu \mathrm{g} \cdot \mathrm{g}^{-1}\right)$, sugerindo que este não se encontra ligado na forma de éter ou éster com outras substâncias. Este valor é 2,5 vezes superior ao resultado encontrado para os AFL da cenoura (46 $\mu \mathrm{g} \cdot \mathrm{g}^{-1}$ ), matriz vegetal de maior concentração desta substância entre as estudadas por Mattila \& Hellström (2007). ${ }^{17}$ Contudo, nota-se que nos AFT este valor diminui, uma vez que ao sofrer hidrólise este ácido pode ter sido degradado. O mesmo ocorre nas matrizes da cenoura, em que não foi detectado o ácido protocatecuico após as hidrólises, com a tangerina $\left(29,64 \mu \mathrm{g} \cdot \mathrm{g}^{-1} \mathrm{de}\right.$
AFL e $6,74 \mu \mathrm{g} \cdot \mathrm{g}^{-1}$ de AFT) e com a laranja bahia (33,52 $\mu \mathrm{g} \cdot \mathrm{g}^{-1}$ de AFL e não detectado nos AFT). ${ }^{17,18}$

$\mathrm{O}$ teor de $p$-hidroxibenzoico $\left(11,43 \mu \mathrm{g} \cdot \mathrm{g}^{-1}\right.$ de AFL e $16,76 \mu \mathrm{g} \cdot \mathrm{g}^{-1}$ de AFT) foi inferior ao de outras matrizes vegetais relatadas na literatura como a tangerina $\left(127,91 \mu \mathrm{g} \cdot \mathrm{g}^{-1} \mathrm{de}\right.$ AFL e $35,23 \mu \mathrm{g} \cdot \mathrm{g}^{-1}$ de AFT), a laranja bahia $\left(193,15 \mu \mathrm{g} \cdot \mathrm{g}^{-1}\right.$ de AFL e 35,99 $\mu \mathrm{g} \cdot \mathrm{g}^{-1}$ de AFT) e a polpa da jussara (34 $\mu \mathrm{g} \cdot \mathrm{g}^{-1}$ de AFL e $540 \mu \mathrm{g} . \mathrm{g}^{-}$ ${ }^{1}$ de AFT). ${ }^{18,19}$

A concentração do ácido $p$-hidroxicinâmico nos AFT $\left(55,00 \mu \mathrm{g} \cdot \mathrm{g}^{-1}\right)$ foi superior ao tomate $\left(10 \mu \mathrm{g} \cdot \mathrm{g}^{-1}\right)$, à polpa de jerivá $\left(21,4 \mu \mathrm{g} \cdot \mathrm{g}^{-1}\right)$ e à polpa de jussara $\left(46 \mu \mathrm{g} \cdot \mathrm{g}^{-1}\right)$, porém é inferior ao rabanete $\left(56 \mu \mathrm{g} \cdot \mathrm{g}^{-1}\right)$, à cenoura $\left(69 \mu \mathrm{g} \cdot \mathrm{g}^{-1}\right)$, 
ao abacate $\left(81 \mu \mathrm{g} \cdot \mathrm{g}^{-1}\right)$ e à tangerina (130 $\mu \mathrm{g} \cdot \mathrm{g}^{-}$ $\left.{ }^{1}\right) .{ }^{17-20}$ Comparando os resultados do ácido $p$ hidroxicinâmico para os arilos do fruto de $C$. fluminensis com a polpa de jerivá, descrito por Martins e colaboradores, pode-se observar que nas duas matrizes este ácido encontra-se predominantemente na forma esterificada, apresentando aumento considerável da concentração após as etapas de hidrólise. ${ }^{20}$

\subsection{Flavonóides}

$\mathrm{Na}$ análise de flavonoides, foram observados dois picos majoritários (Figura 8), sendo o primeiro em um tempo de retenção de $13,88 \mathrm{~min}$ e o segundo de $17,90 \mathrm{~min}$.

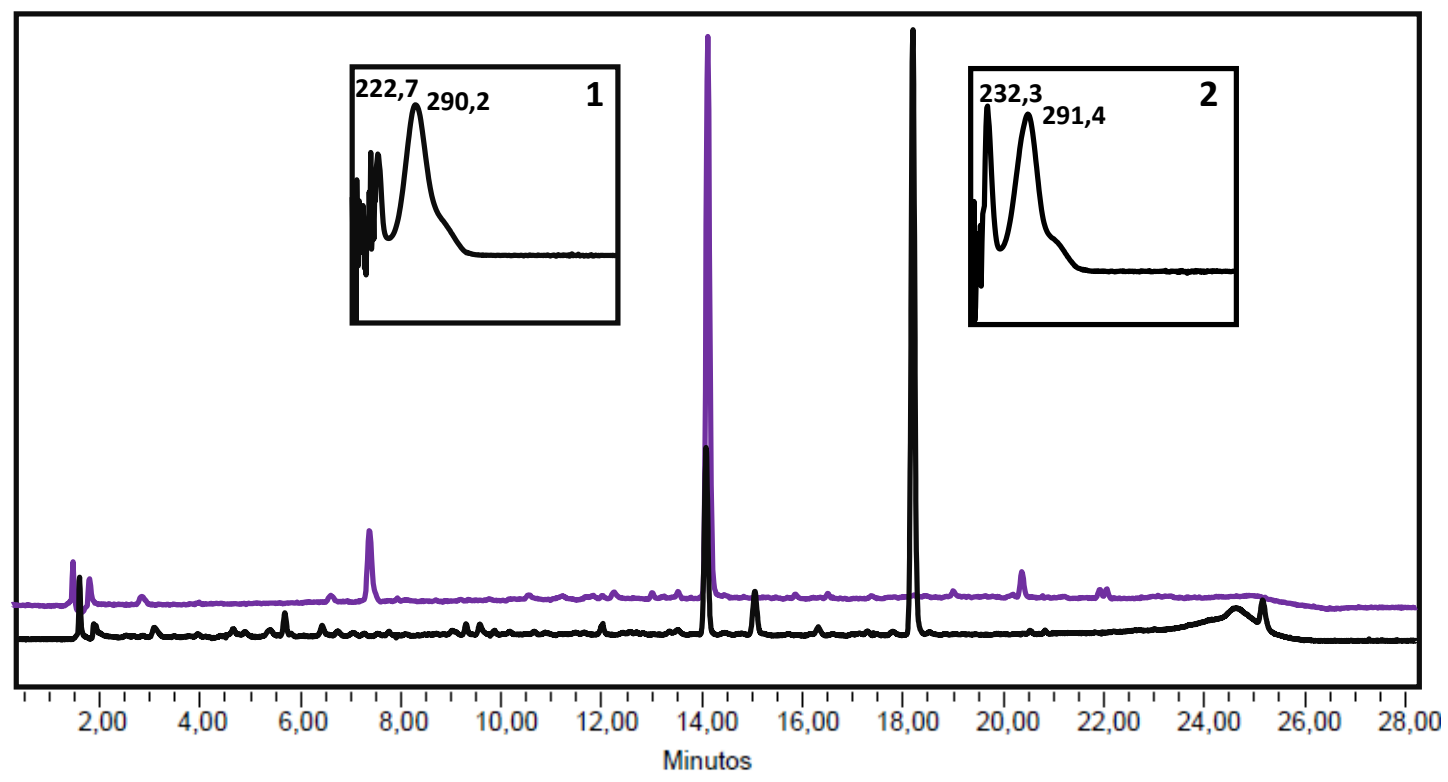

Figura 8. Cromatogramas, por CLAE-DAD, da solução padrão de rhamnetina, em roxo, e do extrato da amostra, em preto, com sobreposição dos picos e espectro de UV referente ao pico da rhamnetina

A comparação dos tempos de retenção e espectros de UV-Vis da mistura de padrões de flavonoides possibilitou a identificação do pico 1 como a rhamnetina metoxiquercetina) (Figura 9).<smiles>COc1cc(O)c2c(=O)c(O)c(-c3ccc(O)c(O)c3)oc2c1</smiles>

Figura 9. Estrutura química da rhamnetina 
O pico majoritário (pico 2), não pôde ser identificado, pois não foi correspondente aos padrões utilizados para comparação, bem como os picos minoritários. Entretanto por apresentar perfil de espectro característico de flavonoide ( 2 bandas de absorção entre $200 \mathrm{e}$ $360 \mathrm{~nm}$ ), e similar ao da rhamnetina, sugerese que o pico 2 seja um derivado deste flavonoide.

\subsection{Carotenoides}

$\mathrm{Na}$ análise de carotenoides (Figura 10) foram detectados picos referentes aos carotenoides-luteína, zeaxantina e $\beta$ criptoxantina (Figura 11).

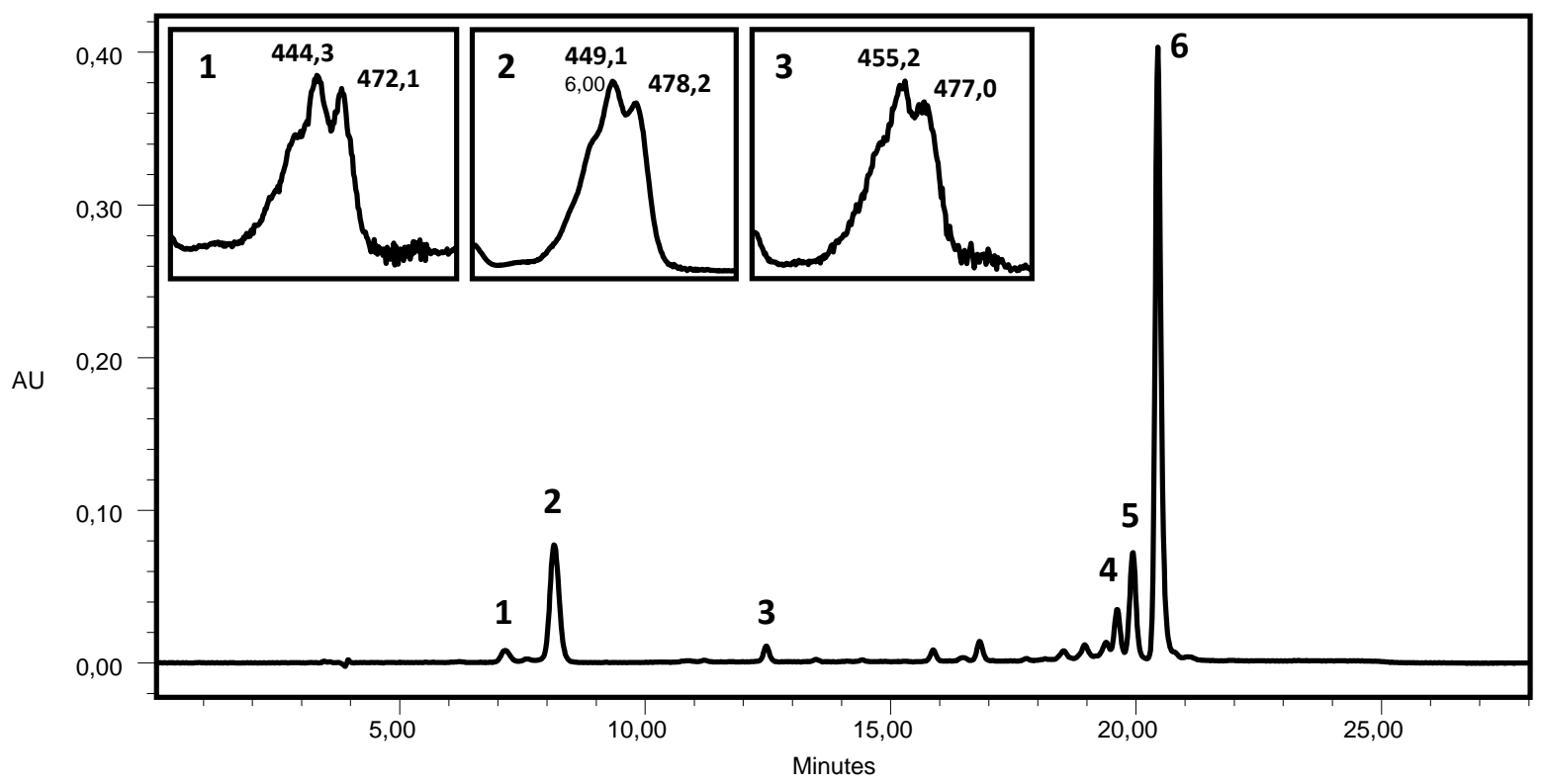

Figura 10. Cromatograma, por CLAE-DAD, do extrato dos arilos de $C$. fluminensis e espectros na região do Visível dos carotenoides identificados: (1) luteína; (2) zeaxantina; (3) $\beta$-criptoxantina; (4), (5) e (6) picos não identificados 
<smiles>CC1=CC(O)CC(C)(C)C1/C=C/C(C)=C/C=C/C(C)=C/C=C/C=C(C)/C=C/C=C(C)/C=C/C1=C(C)CC(O)CC1(C)C</smiles>

Luteína<smiles>CC1=C(/C=C/C(C)=C/C=C/C(C)=C/C=C/C=C(C)/C=C/C=C(C)/C=C/C2=C(C)CC(O)CC2(C)C)C(C)(C)CC(O)C1</smiles>

Zeaxantina<smiles>CC1=C(/C=C/C(C)=C/C=C/C=C/C(C)=C/C=C/C=C(C)/C=C/C=C(C)/C=C/C2=C(C)CC(O)CC2(C)C)C(C)(C)CCC1</smiles>

$\beta$-criptoxantina

Figura 11. Estruturas dos carotenoides presentes nos arilos de $C$. fluminensis

A presença de outros picos não identificados no cromatograma, com $t_{R}$ de aproximadamente $20 \mathrm{~min}$, pode estar relacionada à presença de carotenoides esterificados. Por isso, fez-se necessário a realização de uma reação de saponificação, a fim de se eliminar os interferentes da matriz. ${ }^{14}$ A Figura 12 refere-se ao cromatograma do extrato saponificado, em que foi realizada a quantificação apenas do pico majoritário zeaxantina.

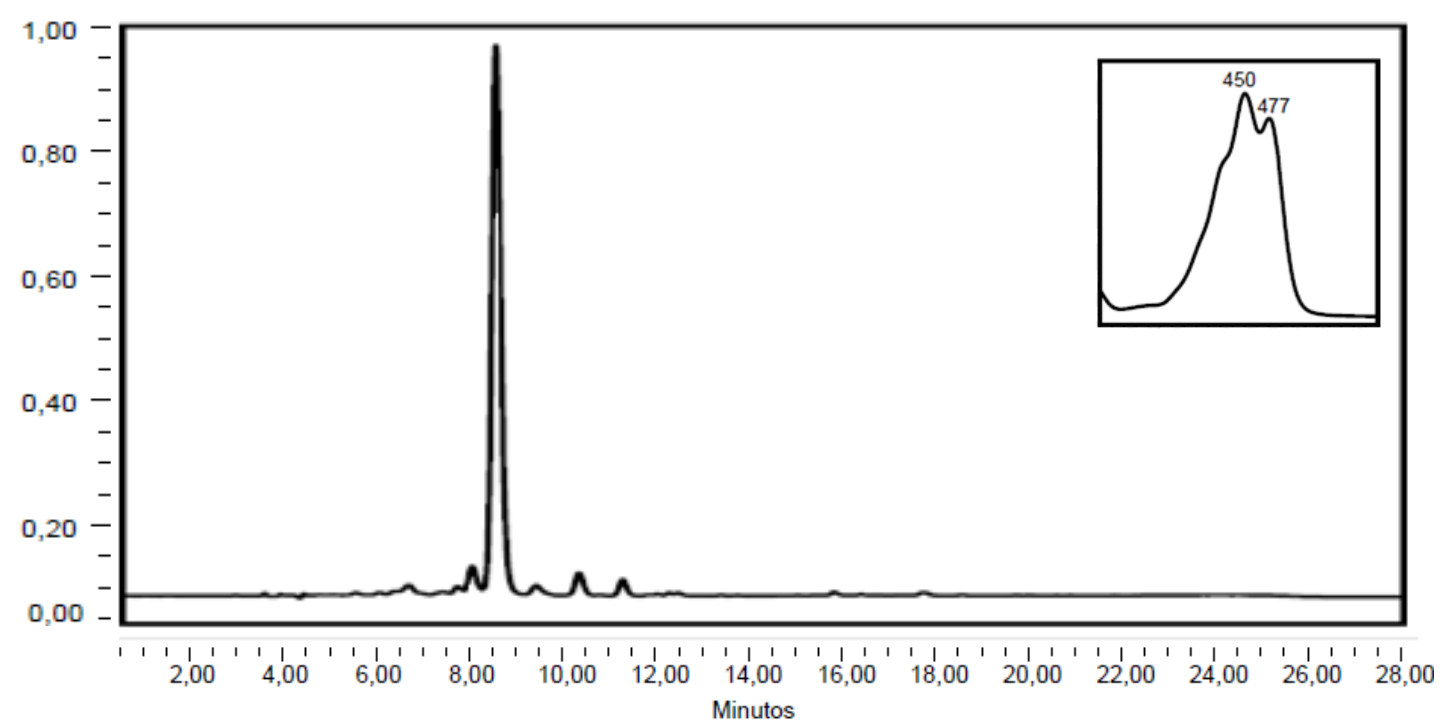

Figura 12. Cromatograma, por CLAE-DAD, do extrato saponificado dos arilos de $C$. fluminensis

$$
\left(t_{R}=8,07 \mathrm{~min}\right)
$$

A equação da reta obtida pela curva 5804,9 , com coeficiente de determinação $\left(r^{2}\right)$ analítica de zeaxantina foi: $y=311706 x-$ de 0,9987 . 
O teor de zeaxantina nos arilos dos frutos de $C$. fluminensis foi de $823 \mu \mathrm{g} . \mathrm{g}^{-1}$, valor este 206 vezes superior ao do milho $\left(4 \mu \mathrm{g} \cdot \mathrm{g}^{-1}\right),{ }^{16}$ que é a principal fonte desse carotenoide na dieta do brasileiro. A zeaxantina apresenta importante função na visão, em razão de conferir a proteção das células nervosas responsáveis pela visão das cores. Diversos estudos epidemiológicos sugerem o importante papel protetor dos carotenoides, luteína e zeaxantina, contra a degeneração macular relacionada à idade e catarata. ${ }^{21,22} \mathrm{~A}$ luteína pode ser encontrada em vegetais verdes escuros. No entanto, a zeaxantina está presente em poucos alimentos e quando presente encontra-se em teores reduzidos, ${ }^{23} \mathrm{O}$ que faz com que seja necessário encontrar uma alternativa para promover a maior ingestão desse carotenoide. Desse modo, identificar novas fontes de zeaxantina pode contribuir para aumentar a disponibilidade de alimentos fontes desse carotenoide.

\section{Conclusão}

A partir do presente trabalho, foi possível a identificação de substâncias bioativas de diferentes classes fitoquímicas nos arilos com sementes dos frutos de $C$. fluminensis. Destacam-se os teores de zeaxantina, superiores aos encontrados em outras matrizes vegetais. Além disso, foram detectados os carotenoides luteína e $\beta$ criptoxantina, os ácidos fenólicos 4hidroxibenzoico, 4-hidroxicinâmico e protocatecuico, e o flavonoide rhamnetina. $\mathrm{O}$ ácido ascórbico (vitamina C) está presente em baixa concentração.

Poucos trabalhos científicos publicados estão relacionados aos frutos da espécie estudada, e abordam apenas os aspectos botânicos e a presença de algumas classes de substâncias. Portanto, os resultados obtidos contribuem para um maior conhecimento sobre a composição e a caracterização de bioativos esta espécie.

\section{Referências Bibliográficas}

${ }^{1}$ Brasil, Governo do Pará, Secretária do Estado do Meio Ambiente. Lei Estadual № 6194 de 12 de janeiro de 1999. [Link]

${ }^{2}$ Pereira, A. P.; Putzke, J.; Dicionário Brasileiro de Botânica, CRV: Curitiba-PR, 2010.

${ }^{3}$ Silva, M. C. A.; Paiva, S. R. Antioxidant activity and flavonoid content of Clusia fluminensis Planch. \& Triana. Anais da Academia Brasileira de Ciências 2012, 84, 609. [CrossRef]

${ }^{4}$ Bittrich, V.; Trad, R. J.; Cabral, F.; NascimentoJr, J. E., Clusiaceae in Lista de Espécies da Flora do Brasil - Jardim Botânico do Rio de Janeiro. Disponível em: $<$ http://reflora.jbri.gov.br/jabot/floradobrasil /FB6834>. Acesso em: 01 julho 2016.

${ }^{5}$ Correia, M. C. R.; Lima, H. A. SILVA, R. C. P. Caracterização dos frutos, sementes e plântulas de espécies de Clusiaceae das restingas do Rio de Janeiro. Rodriguésia Revista do Jardim Botânico do Rio de Janeiro 2013, 64, 61. [CrossRef]

${ }^{6}$ Sítio do Ministério do Meio Ambiente - Brasil. Disponível em: $<$ http://www.mma.gov.br/biodiversidade/bio diversidade-brasileira $>$. Acesso em: 01 julho 2016.

7 Collins, C. H.; Braga, G. L.; Bonato, P. S. Fundamentos da cromatografia, Editora da UNICAMP: Campinas-SP, 2007.

8 Cunha, C. P; Dissertação de Mestrado, Universidade Federal Rural do Rio de Janeiro, 2013. [Link]

9 Silva, M. C. A; Dissertação de Mestrado, Universidade Federal Fluminense, 2011.

${ }^{10}$ Pacheco, S.; Godoy, R. L. O.; Peixoto, F. M.; Gouvêa, A. C. M. S.; Santiago, M. C. P. A.; Felberg, I.; Borguini, R. G. Preparation of high purity analytical standards using high performance liquid chromatography in analytical scale. Analytical Chemistry - An Indian Journal 2013, 12, 194. [Link]

${ }^{11}$ Rosa, J. S.; Godoy, R. L. O.; Oiano Neto, J.; Campos, R. S.; Matta, V. M.; Freire, C. A.; Silva, 
A. S.; Souza, R. S. Desenvolvimento de um método de análise de vitamina $\mathrm{C}$ em alimentos por cromatografia líquida de alta eficiência e exclusão iônica. Ciência $e$ Tecnologia de Alimentos 2007, 27, 837. [CrossRef]

12 Mattila, P.; Kumpulainen, J. Determination of Free and Total Phenolic Acids in PlantDerived Foods by HPLC with Diode-Array Detection. Journal of Agricultural and Food Chemistry 2002, 50, 3660. [CrossRef] [PubMed]

${ }^{13}$ Klump, S. P.; Alfred, M. C.; Macdonald, J. L. Determination of isoflavones in soy and selected foods containing soy by extraction, saponification, and liquid chromatography: Collaborative study. Journal of $A O A C$ International 2001, 84, 1865. [PubMed]

14 Rodríguez-Amaya, D. B. A Guide to Carotenoid Analysis in Foods, 2001. [Link]

${ }^{15}$ Pacheco, S.; Peixoto, F. M.; Borguini, R. G.; Nascimento, L. S. M.; Bobeda, C. R. R.; Santiago, M. C. P. A.; Godoy, R. L. O. Microscale extraction method for HPLC carotenoid analysis in vegetable matrices. Scientia Agricola 2014, 71, 416. [CrossRef]

16 Tabela Brasileira de Composição de Alimentos. 4a ed. NEPA - Unicamp: Campinas, 2011. [Link]

${ }^{17}$ Mattila, P.; Hellström, J. Phenolic acids in potatoes, vegetables, and some of their products. Journal of Food Composition and Analysis 2007, 20, 152. [CrossRef]

${ }^{18}$ Sun, Y.; Qiao, L.; Shen, Y.; Jiang, P.; Chen, J.; $\mathrm{Ye}, \mathrm{X}$. Phytochemical profile and antioxidant activity of physiological drop of citrus fruits. Journal of food science 2013, 78, C37. [CrossRef]

${ }^{19}$ Inada, K. O. P.; Oliveira, A. A.; Revorêdo, T. B.; Martins, A. B. N.; Lacerda, E. C. Q.; Freire, A. S.; Braz, B. F.; Santelli, R. E.; Torres, A. G.; Perrone, D.; Monteiro, M. C. Screening of the chemical composition and occurring antioxidants in jabuticaba (Myrciaria jaboticaba) and jussara (Euterpe edulis) fruits and their fractions. Journal of Functional Foods 2015, 17, 422. [CrossRef]
${ }^{20}$ Martins, V. C.; Braga, E. C. O.; Mazza, K. E. L.; Rocha, J. F.; Cunha, C. P.; Pacheco, S.; Nascimento, L. S. M.; Santiago, M. C. P. A.; Borguini, R. G.; Godoy, R. L. O. Caracterização química da polpa do fruto jerivá (Syagrus romanzoffiana Cham.). Revista Virtual de Química 2015, 7, 2422. [CrossRef]

${ }^{21}$ Trieschmann, M.; Beatty, S.; Nolan, J. M.; Hense, H. W.; Heimes, B.; Austermann, U.; Fobker, M.; Pauleikhoff, D. Changes in macular pigment optical density and serum concentrations of its constituent carotenoids following supplemental lutein and zeaxanthin: The LUNA study. Experimental Eye Research 2007, 84, 718728. [CrossRef]

22 Delcourt, C.; Carrie'Re, I.; Delage, M.; Barberger-Gateau, P.; Schalch, W. Plasma Lutein and Zeaxanthin and Other Carotenoids as Modifiable Risk Factors for AgeRelated Maculopathy and Cataract: The POLA Study. Investigative Ophthalmology \& Visual Science 2006, 47, 2329. [CrossRef]

23 Rodriguez-Amaya, D. B.; Kimura, M.; Amayafarfan, J. Fontes brasileiras de carotenoides: tabela brasileira de composição de carotenoides em alimentos. Brasília: Ministério do Meio Ambiente / Secretaria de Biodiversidade e Floresta, 2008. [Link] 